\author{
Articulo original \\ (Original paper)
}

\title{
ASPECTOS ECOLÓGICOS DE UNA COMUNIDAD DE ANUROS EN UN AMBIENTE TROPICAL ESTACIONAL EN GUANAJUATO, MÉXICO
}

\section{ECOLOGICAL ASPECTS OF AN ANURAN COMMUNITY IN A SEASONAL TROPICAL ENVIRONMENT IN GUANAJUATO, MEXICO}

\author{
ADRIAN LEYTE-MANRIQUE ${ }^{*}$, RUTH LILIANA E. GONZÁLEZ-GARCÍA ${ }^{2}$, GUSTAVO ERNESTO \\ QUINTERO-DÍAZ ${ }^{3}$, FRANCISCO ALEJO-ITURVIDE ${ }^{2}$, CHRISTIAN BERRIOZABAL-ISLAS ${ }^{4}$ \\ ${ }^{1}$ Laboratorio de Biología, Tecnológico Nacional de México, Campus Salvatierra (ITESS). Manuel Gómez Morín 300, Col. \\ Janicho, Salvatierra, Guanajuato, CP 38933, México. <aleyteman@gmail.com> \\ ${ }^{2}$ Instituto Tecnológico Superior de Irapuato, Km. 12.5 s/n, carretera Irapuato-Silao, El Copal, Irapuato, Guanajuato, CP 36821, \\ México.<rlgg1@outlook.com>; <fralejo04@gmail.com> \\ ${ }^{3}$ Departamento de Biología, Centro de Ciencias Básicas, Universidad Autónoma de Aguascalientes. Av. Universidad 940, \\ Aguascalientes, Ags., CP 20131, México. <gequintmxags@hotmail.com> \\ ${ }^{4}$ Laboratorio de Ecología del Paisaje, Centro de Investigaciones Biológicas, Universidad Autónoma del Estado de Hidalgo, \\ Pachuca, Hidalgo, 1-69 Plaza Juárez, CP 42001, México. <christianberriozabal@gmail.com> \\ *Autor de correspondencia: <aleyteman@gmail.com>
}

Recibido: 20/02/2018; aceptado: 18/09/2018; publicado en línea: 16/11/2018

Editor responsable: Gustavo Aguirre

Leyte-Manrique, A., González-García, R. L. E., Quintero-Díaz, G. E., Alejo-Iturvide, F., Berriozabal-Islas, C. (2018) Aspectos ecológicos de una comunidad de anuros en un ambiente tropical estacional en Guanajuato, México. Acta Zoológica Mexicana (nueva serie), 34, 1-14. https://doi.org/10.21829/azm.2018.3412138

RESUMEN. En este estudio comparamos aspectos ecológicos del uso del microhábitat y periodo de actividad de una comunidad de anuros en cuatro tipos de vegetación (bosque tropical caducifolio, bosque de galera, matorral xerófilo y pastizal inducido) en un ambiente tropical estacional de la región suroeste del estado de Guanajuato, en el Área Natural Protegida Las Musas. Se registraron nueve especies de anuros, tres de sapos y seis de ranas, de las cuales la más abundante fue Lithobates neovolcanicus (152 individuos), y las menos abundantes, con un solo individuo Craugastor occidentalis y Spea multiplicata. Se caracterizaron 17 distintos tipos de microhábitats, con diferencias entre especies $(H=0.22 ; P=0.01)$ en cuanto a su uso. Los tipos de vegetación con la mayor heterogeneidad de microhábitats fueron el bosque tropical y el bosque de galería, en comparación con el matorral xerófilo y pastizal. Las especies que usaron más microhábitats fueron L. neovolcanicus, Anaxyrus compactilis y Dryophytes eximius. El mayor solapamiento se registró entre A. compactilis e Incilius occidentalis con el $100 \%$; para $I$. occidentalis y D. arenicolor fue de $89 \%$ y para I. occidentalis y L. neovolcanicus de $72 \%$. Los periodos de actividad más prolongados se registraron en A. compactilis (10:28 a 22:10 hrs) y L. neovolcanicus (10:40 a 22:20 hrs). Se encontró una relación positiva entre la temperatura corporal y la temperatura del microhábitat y la ambiental para A. compactilis, I. occidentalis, D. eximius, D. arenicolor, y $L$. neovolcanicus. La comunidad de anuros presente en el área de estudio puede diferenciarse en tres componentes eco-estructurales, conforme a sus hábitos; especies acuáticas, saxícolas-terrestres y arborícolas, determinados tanto por sus hábitos para la selección de microhábitats, como por sus periodos de actividad diarios. Consideramos que si bien, la heterogeneidad vegetal es importante en la distribución de las especies y disponibilidad de microhábitats, no es el principal factor en la selección y uso de los 
microhábitats por parte de las especies como se suponía. Sino que la selección y uso de los microhábitats y composición de la comunidad de anuros, se determinó por eventos conductuales que se manifiestan en los periodos de actividad y capacidad de termorregular de cada especie, como una respuesta de orden fisiológico a la influencia de la temperatura.

Palabras clave: Microhábitat, actividad, anuros, comunidad, Las Musas, Guanajuato.

Leyte-Manrique, A., González-García, R. L. E., Quintero-Díaz, G. E., Alejo-Iturvide, F., Berriozabal-Islas, C. (2018) Ecological aspects of an anuran community in a seasonal tropical environment in Guanajuato, Mexico. Acta Zoológica Mexicana (nueva serie), 34, 1-14. https://doi.org/10.21829/azm.2018.3412138

\begin{abstract}
In this study we compared ecological aspects of the use of the microhabitat and period of activity of an anuran community in four types of vegetation (deciduous tropical forest, gallery forest, xerophilous scrub and induced pasture) in a seasonal tropical environment of the southwestern region of the state of Guanajuato, Las Musas Protected Natural Area. There were nine species of anurans, three toads and six frogs, of which the most abundant was Lithobates neovolcanicus (152 individuals), and the least abundant, with only one individual Craugastor occidentalis and Spea multiplicata. We characterized 17 different types of microhabitats, differences between species $(H=0.22, P=0.01)$ exist in terms of their use. The vegetation types with the greatest heterogeneity of microhabitats were the low forest and the gallery forest in comparison with the xerophilous and grassland scrub. Species that used most microhabitats were L. neovolcanicus, Anaxyrus compactilis and Dryophytes eximius. The highest overlap was recorded between A. compactilis and Incilius occidentalis (100\%), followed by I. occidentalis and $D$. arenicolor (89\%), and I. occidentalis and L. neovolcanicus (72\%). The longest periods of activity were recorded in A. compactilis (10:28 to 22:10 hrs) and L. neovolcanicus (10:40 to 22:20 hrs). A positive relationship was found between the body temperature and temperature of the microhabitat and the environmental one for $A$. compactilis, $I$. occidentalis, $D$. eximius, $D$. arenicolor and $L$. neovolcanicus. The community of anurans present in the study area can be differentiated into three eco-structural components, according to their habits; aquatic, saxicolous-terrestrial and arboreal species, determined, both by their habits for the selection of microhabitats, and their daily activity periods. We consider that although plant heterogeneity is important in the distribution of species and availability of microhabitats, it is not the main factor in the selection and use of microhabitats by species as was supposed. But the selection and use of the microhabitats and composition of the community of anurans, is determined by behavioral events that are manifested in the periods of activity and thermoregulatory capacity of each species, as a physiological response to the influence of the temperature.
\end{abstract}

Key words: Microhabitat, activity, anurans, community, Las Musas, Guanajuato.

\title{
INTRODUCCIÓN
}

La relación entre los organismos y su ambiente se aborda desde varias perspectivas de estudio, una de ellas, las interacciones ecológicas como promotoras de la complejidad estructural de las comunidades y en los valores de riqueza y diversidad en éstas (Krebs, 1999; Chave, 2013; Soto-Sandoval et al., 2017). Esta relación entre diversidad y funcionalidad se encuentra estrechamente en relación con factores de tipo biológico, fisiográfico, climático, ecológico y de distribución, a los cuales responden los seres vivos (Maciel-Mata et al., 2015). En este contexto, organismos como los anfibios responden con variadas estrategias reproductivas, fisiológicas y conductuales encaminadas a la selección y uso de los recursos, entre los que destacan el microhábitat y el alimento (Acuña-Vargas, 2016), y de manera intrínseca con sus 
periodos de actividad diarios y estacionales (Vitt \& Caldwell, 2014; Williams-León de Castro \& ReySánchez, 2014; Luja et al., 2016).

En ambientes estacionales, sea el caso de los bosques tropicales caducifolios, los factores que inciden sobre la actividad y ocurrencia de los anuros son la precipitación y humedad, así como la disponibilidad de refugios y alimento que explotan estos y que puede definir su abundancia, estructura y distribución espacial dentro de la matriz paisajística (Pounds et al., 1999; Muñoz-Guerrero et al., 2007; Blanco-Torres, 2009; Suazo-Ortuño et al., 2015). En relación al uso del microhábitat se documenta en ambientes tropicales que el uso de éstos, por parte de los anuros depende de la heterogeneidad del hábitat y la actividad de las especies, con ello ocurre un solapamiento entre especies (Vasconcelos \& Rosas-Feres, 2008; Soto-Sandoval et al., 2017). Por ejemplo, Juárez-Ortiz et al. (2016) en un bosque tropical subcaducifolio en Sierra Negra, Puebla analizaron la vocalización de un ensamble de machos y su relación con parámetros ambientales como la altura de la percha y el uso de microhábitat, encontraron variaciones tanto de la hora de actividad, microhábitat en uso, percha y frecuencia de vocalización. Finalmente, cabe señalar que los anuros son sensibles a los cambios de su ambiente, cambios que pueden modificar drásticamente sus relaciones ecológicas, biología reproductiva, diversidad y abundancia, la cual está estrechamente relacionada a sus hábitos y microhábitats que explotan (Lips et al., 2004). Por ello un ambiente tropical como el que se localiza en el Área Natural Protegida Las Musas, es el sitio de estudio que se eligió para conocer y generar información concerniente a aspectos ecológicos del uso del microhábitat y actividad de los anuros en ambientes tropicales estacionales en el estado de Guanajuato. Se añade a ello, que para grupos como el de los anuros no se cuenta en la entidad con información referente a su ecología (Leyte-Manrique et al., 2016). Si bien, el sitio de estudio, Las Musas, se inserta en un ambiente tropical con marcada estacionalidad, se espera que la heterogeneidad de sus componentes vegetales permita vislumbrar diferencias no sólo estructurales en la comunidad de anuros sino también en el uso del microhábitat y periodos de actividad. Por lo que se asume que la heterogeneidad vegetal puede ser un factor de respuesta a la selección del microhábitat y actividad de las especies. Así, el objetivo de este estudio consistió en caracterizar los microhábitats que usan las especies y ver si existe un solapamiento de nicho (microhábitat), así como determinar los periodos de actividad entre especies.

\section{MATERIALES Y MÉTODOS}

Área de estudio. El Área Natural Protegida Las Musas (ANPLM), tiene una extensión de 3,174.76 ha y se cataloga como área de uso sustentable. Se localiza en el municipio de Manuel Doblado, estado de

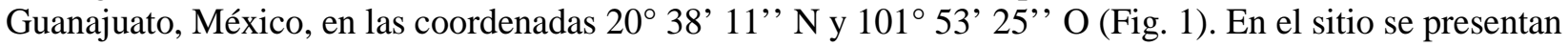
elevaciones que van de los 1741 a $1810 \mathrm{msnm}$. El clima corresponde al subtropical estacional con una precipitación y temperatura promedio anual de $787 \mathrm{~mm}$ y $20^{\circ} \mathrm{C}$, con una máxima de $38.5^{\circ}$ y mínima de $2.6^{\circ} \mathrm{C}$, respectivamente (García, 1973). Su principal afluente es el río Colorado, el cual se extiende a lo largo de toda el ANP (Walter \& Brooks, 2009). Los tipos de vegetación presentes en el sitio son el, bosque tropical caducifolio (BTC), bosque de galera (BG), matorral xerófilo (MX), y pastizal inducido (P) (Rzedowski, 1978; Fig. 2).

De julio de 2015 a junio de 2016 se realizaron muestreos sistemáticos en el sitio de estudio, de manera mensual y con una duración de dos días, cubriéndose un total de 12 salidas. El esfuerzo de muestreo fue de 12 horas/hombre; cuatro horas por la mañana (7:00-11:00), tarde (14:00-18:00) y noche (19:00-23:00 hrs) con el fin de cubrir las principales horas de actividad de los anuros (Pérez-Tobar, 2011; Luria-Manzano, 2012). Se usó la técnica de muestreo por transectos de 10 x 300 m y con una separación entre éstos de $50 \mathrm{~m}$ para cada tipo de vegetación con la finalidad de tener independencia de los datos (Vite-Silva et al., 2010). Se llevó a cabo una búsqueda directa de los organismos, la cual se complementó con un relevamiento por encuentros visuales (Vargas \& Bolaños, 1999), tomando en consideración los hábitos y actividad de los anuros. Durante la colecta nocturna se emplearon lámparas de cabeza para la 


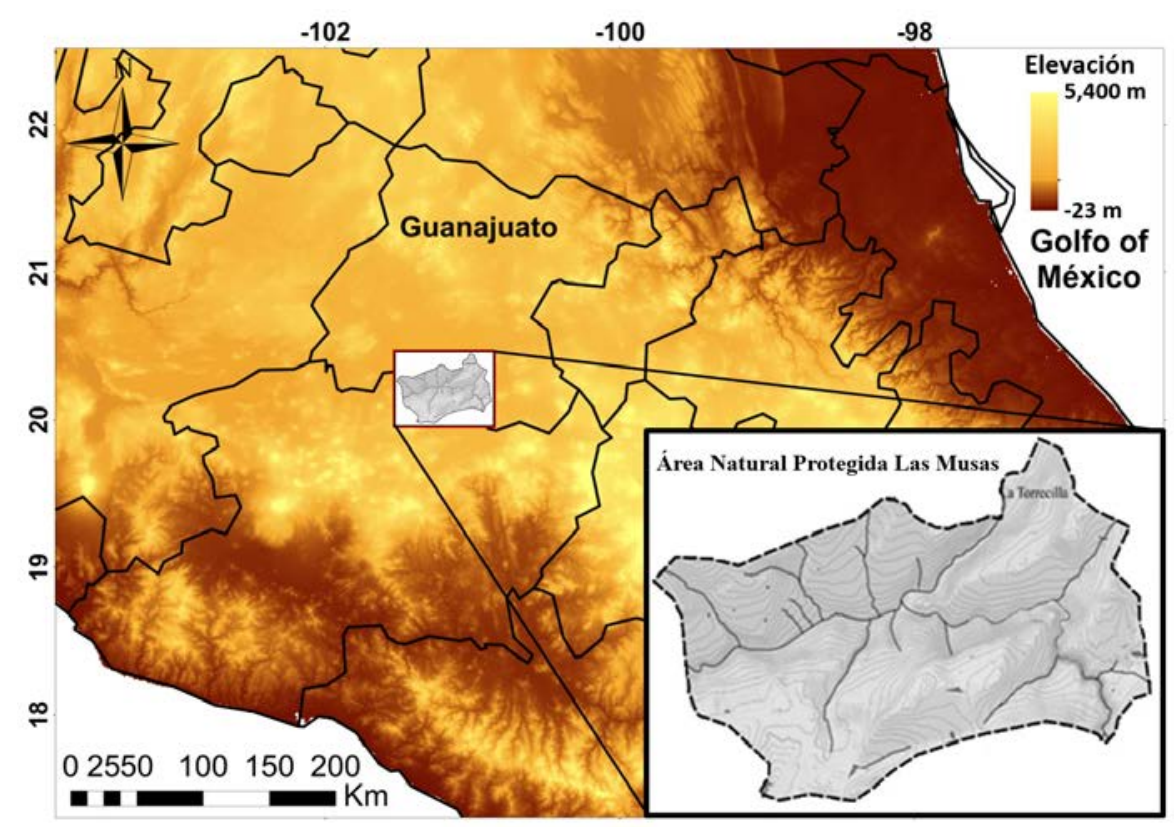

Figura 1. Mapa del área de estudio. Área Natural Protegida Las Musas, Manuel Doblado, Guanajuato.

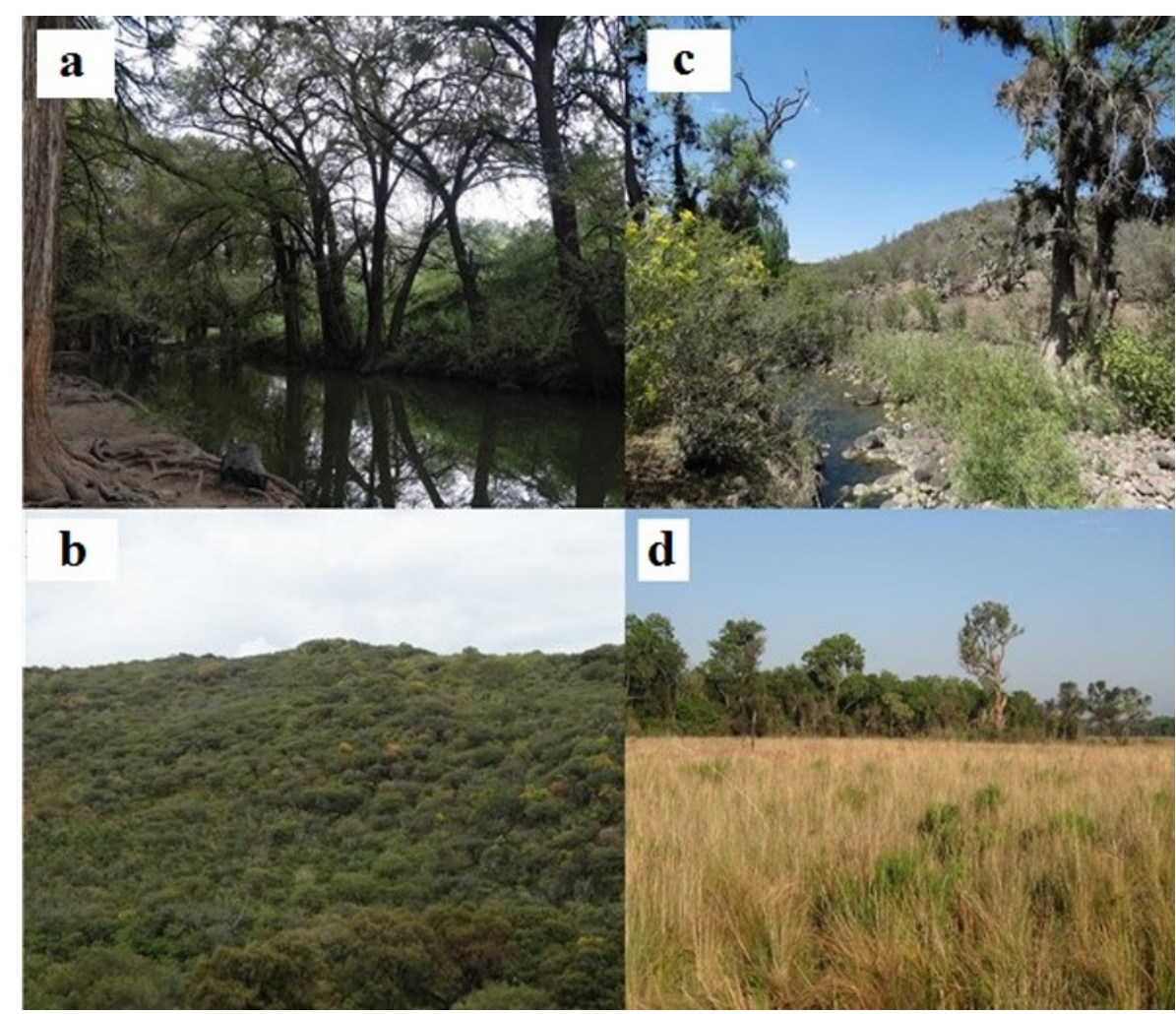

Figura 2. Tipos de vegetación representativos en el Área Natural Protegida Las Musas, Guanajuato: a = Bosque de galería, $\mathrm{b}=$ Bosque tropical caducifolio, $\mathrm{c}=$ Matorral xerófilo y d $=$ Pastizal inducido. 
localización de los organismos. Estos se buscaron en cuerpos de agua como son arroyos y pozas, entre la vegetación, rocas y ramas circundantes. La captura de los organismos se llevó a cabo con ayuda de redes tipo entomológico o directamente con la mano, según técnicas convencionales propuestas por CasasAndreu et al. (1991).

A cada organismo se le tomaron datos de LHC (longitud hocico cloaca en mm), y temperatura cloacal; adicionalmente se tomaron datos de la temperatura ambiental y del microhábitat. La lectura se hizo con un termómetro digital con capacidad de -10 a $80^{\circ} \mathrm{C}$. Así mismo, se registraron datos de la hora de avistamiento, tipo de microhábitat, tipo de vegetación y coordenadas de ubicación geográfica. Los organismos se identificaron in situ con apoyo de guías especializadas para anfibios (ej. Vázquez-Díaz \& Quintero-Díaz, 2005; Ramírez-Bautista et al., 2009). Posteriormente, se fotografiaron para generar un acervo de imágenes y se liberaron en el lugar de captura.

Uso del microhábitat y actividad. Los hábitos de los anuros se caracterizaron en tres tipos: acuáticos, saxícolas-terrestres y arborícolas de acuerdo a la propuesta de García et al. (2016), la cual consistió en identificar los distintos microhábitats terrestres y acuáticos en los que se encontraban los anuros, por ejemplo: pozas de agua, arbustos y rocas, entre otros. Una vez identificados se codificaron y se les asignó un valor numérico para el análisis estadístico. La actividad de los anuros se determinó conforme a sus horas de actividad durante el día o la noche.

Análisis de datos. Uso del microhábitat. Para determinar si existían diferencias entre especies con respecto al tipo de microhábitat, se desarrolló una prueba de ANOVA no paramétrica de Kruskal-Wallis, con un factor de respuesta a las especies y como variable independiente los microhábitats. Los resultados de la relación uso del microhábitat por tipos de vegetación fueron interpretados a partir de diagramas de perfiles multivariados, ya que son una herramienta útil para interpretar las relaciones conjuntas de las variables sobre un factor de respuesta esperado (Gabriel, 1971). Se determinó la sobreposición de nicho (microhábitat) entre especies a partir del índice de solapamiento de nicho de Pianka (1986); Ojk $=\Sigma \mathrm{i}$ PijPik / $\Sigma$ i Pij $\Sigma$ i; dónde: Ojk = Medida de Pianka para la sobreposición de nicho entre la especie j y la especie $\mathrm{k}$ (j y k), Pij = Proporción del recurso i es el total de recursos usados por la especie $\mathrm{j}$, Pik = Proporción del recurso i, es el total de los recursos usados por la especie $k, n=$ Número total de recursos (para este caso son los tipos de microhábitats). Los valores de este índice varían entre 0 (sin solapamiento) y 1 (solapamiento total en el recurso i). El análisis de solapamiento de nicho se efectuó con apoyo del programa Ecological Methodology, Versión 2 (Krebs, 1999).

Actividad. Para expresar diferencias en cuanto a la hora de actividad entre especies, se efectuó un ANOVA no paramétrico de Kruskal-Wallis (como variable dependiente se usó la hora de actividad, y como regresora, a las especies). Para ver las diferencias entre medias de tratamientos se realizó una prueba de $\mathrm{F}$ de igualdad de varianza (Zar, 1999). Cabe señalar que para el análisis de los datos solo se consideraron aquellas especies que se encontraron activas al momento de su captura, aquellas que se encontraban debajo de rocas, sustratos y troncos, no se consideraron para el análisis. Para determinar si existía relación entre la temperatura corporal y las temperaturas del microhábitat y ambiental, con respecto a la actividad de las especies se desarrolló un análisis de regresión lineal. Para los análisis de actividad y relación entre temperatura corporal y del microhábitat se consideró un tamaño de muestra de $\mathrm{N}=209$ individuos: A. compactilis $(\mathrm{n}=50)$, I. occidentalis $(\mathrm{n}=5), D$. arenicolor $(\mathrm{n}=16), D$. eximius $(\mathrm{n}=56), H$. variolosus $(\mathrm{n}=10)$, y L. neovolcanicus $(\mathrm{n}=72)$. En el caso de $S$. multiplicata $(\mathrm{n}=1)$, C. occidentalis $(\mathrm{n}=$ 1) y E. guttilatus $(\mathrm{n}=2)$, no se consideraron para el análisis de regresión lineal, dado que el tamaño de muestra no era estadísticamente representativo.

Finalmente mediante un análisis de componentes principales (ACP) se analizó la relación y variación entre especies en función del uso del microhábitat y actividad que explique la estructura de la comunidad de anuros. Las variables a relacionar fueron: Microhábitat $=\mathrm{MH}$, temperatura del microhábitat 
$=\mathrm{T}^{\circ} \mathrm{CMH}$, temperatura ambiental $=\mathrm{T}^{\circ} \mathrm{CA}$, vegetación $=$ VEG, hora de actividad $=$ HORA, y temperatura cloacal $=\mathrm{T}^{\circ} \mathrm{CC}$. Los datos se ajustaron y normalizaron a log base 10 a fin de que las correlaciones fueran lineales, al ser los datos de las variables de distinta magnitud escalar (Balzarini et al., 2015). Los resultados que se obtuvieron en este trabajo se expresan con un valor medio \pm E.E (Error estándar), y se utilizó el programa estadístico InfoStat versión 2.1.

\section{RESULTADOS}

Después de 12 muestreos de campo se registró una abundancia de 496 organismos, de siete familias, ocho géneros y nueve especies de anuros para el ANPLM. Las especies más abundantes fueron $L$. neovolcanicus, D. eximius, A. compactilis, I. occidentalis e $H$. variolosus. En tanto que las menos abundantes fueron E. gutiilatus, C. occidentalis y S. multiplicata, con dos y un individuo, respectivamente (Cuadro 1). En cuanto a la abundancia por mes y estación, la rana L. neovolcanicus tuvo la mayor abundancia para los meses de julio, agosto y septiembre, seguida de $D$. eximius en los meses de abril a septiembre (lluvias) y en abril (secas). Especies como H. variolosus (10 individuos), E. guttilatus (2), C. occidentalis (1) y S. multiplicata (1), sólo se observaron en la estación de lluvias (Fig. 3).

Cuadro 1. Abundancia proporcional de las especies de la comunidad de anuros en el Área Natural Protegida Las Musas, Guanajuato. LHC $(\mathrm{mm})$ = promedio e intervalos de longitud hocico-cloaca.

\begin{tabular}{ccccc}
\hline Familia & Género & Especie & Abundancia & LHC \\
\hline Bufonidae & Anaxyrus & compactilis & 135 & $40.8(17.7-54)$ \\
& Incilius & occidentalis & 16 & $37.2(33.1-42.5)$ \\
Scaphiopodidae & Spea & multiplicata & 1 & 34.7 \\
Craugastoridae & Craugastor & occidentalis & 1 & 35 \\
Eleutherodactylidae & Eleutherodactylus & guttilatus & 2 & $39.5(23.2-55.9)$ \\
Hylidae & Dryophytes & arenicolor & 35 & $30(19.8-45.6)$ \\
& Dryophytes & eximius & 144 & $56.2(23.2-58)$ \\
Microhylidae & Hypopachus & variolosus & 10 & $33.6(29.3-39.7)$ \\
Ranidae & Lithobates & neovolcanicus & 152 & $29.6(14.4-72)$ \\
\hline $\boldsymbol{N}$ & & & $\mathbf{4 9 6}$ \\
\hline
\end{tabular}
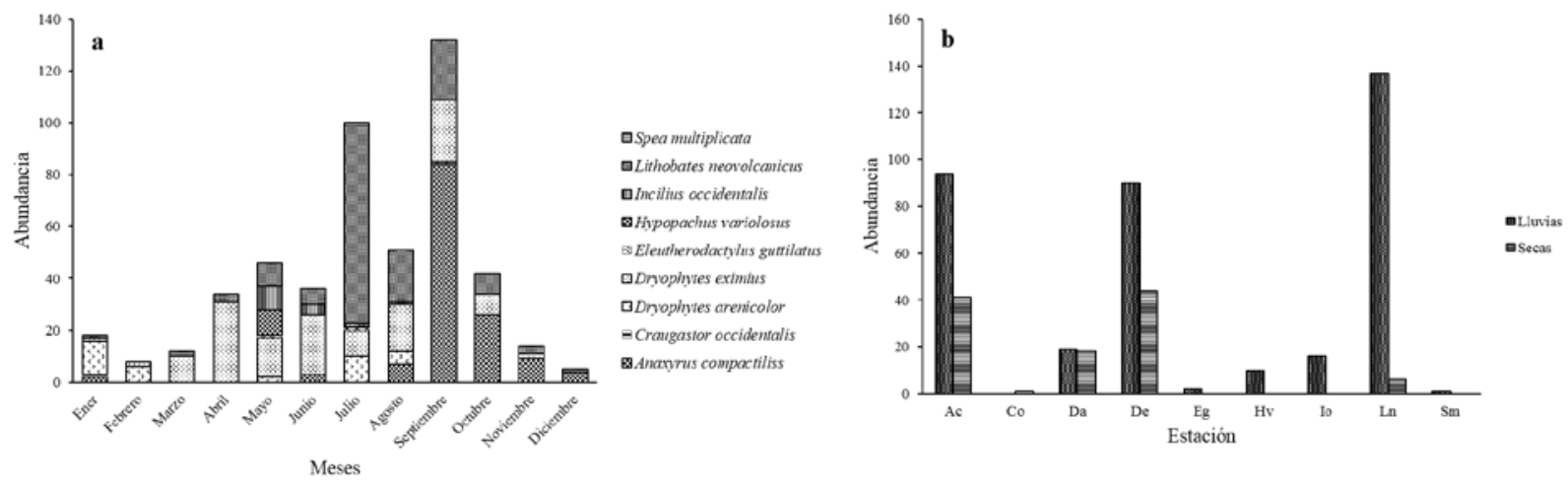

Figura 3. Abundancia por mes y estación (secas y lluvias) de las especies de anuros en el Área Natural Protegida Las Musas, Guanajuato. Especies; Ac = Anaxyrus occidentalis, Co = Craugastor occidentalis, $\mathrm{Da}=$ Dryophytes arenicolor, $\mathrm{De}=D$. eximius, $\mathrm{Eg}=$ Eleutherodactylus guttilatus, $\mathrm{Hv}=$ Hypopachus variolosus, Io = Incilius occidentalis y Ln = Lithobates neovolcanicus y Sm= Spea multiplicata . 

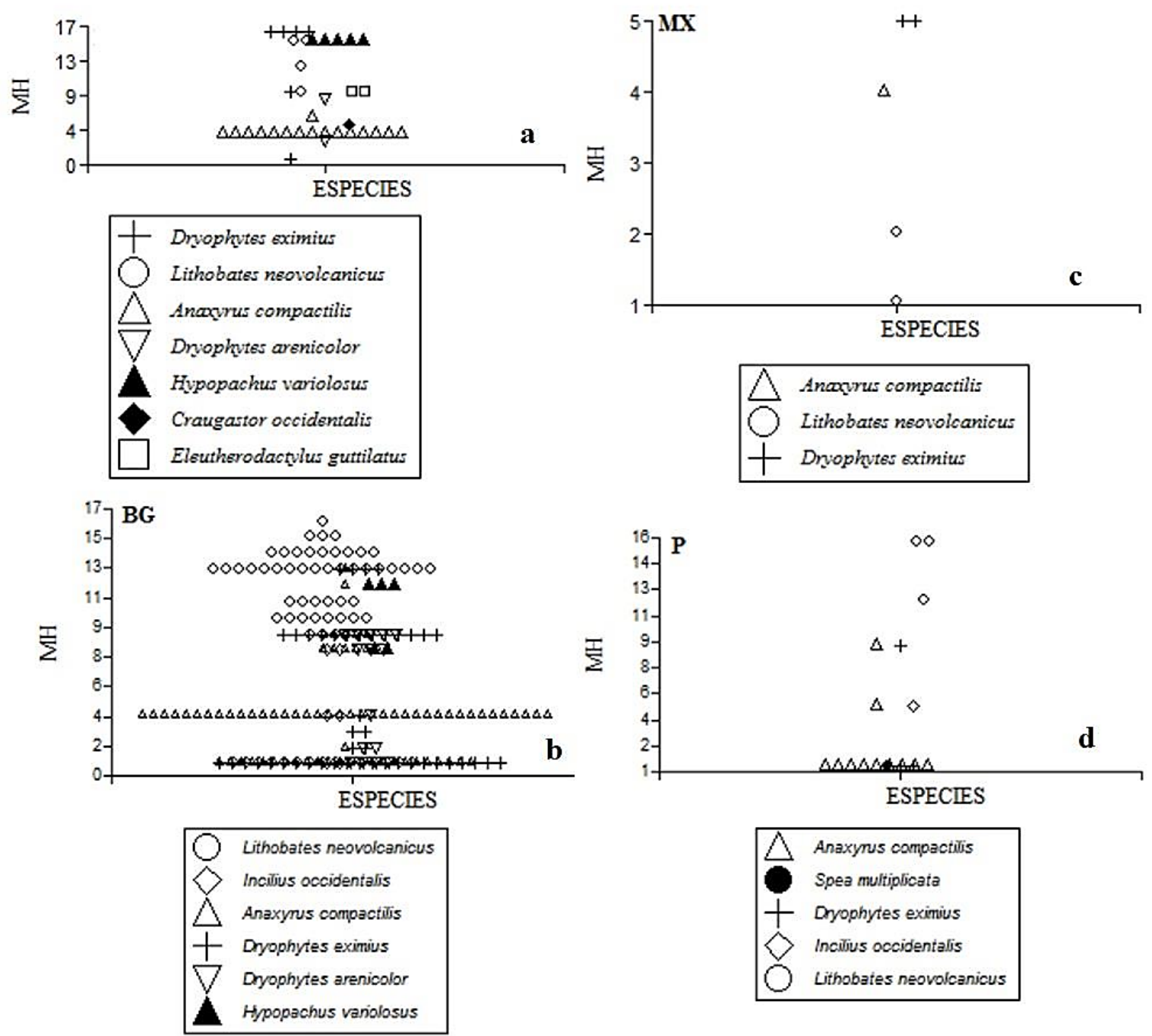

Figura 4. Diagramas de perfiles que muestran los microhábitats utilizados por tipo de vegetación por las especies de la comunidad de anuros en el Área Natural Protegida Las Musas, Guanajuato.

Microhábitats. Para este estudio se identificaron 17 microhábitats (Sobre pasto $=1$, en hojarasca $=2$, sobre tierra $=3$, en capa asfáltica $=4$, bajo roca $=5$, grieta en roca $=6$, sobre roca $=7$, entre vegetación $=8$, en arbusto $=9$, sobre tronco $=10$, sobre roca en río $=11$, en charca de agua $=12$, sobre lecho de río $=13$, en contenedor de agua $=14$, en río $=15$, en canaleta $=16$, $\mathrm{y}$ en poza y/o charca de agua = 17), los cuales de manera general se agruparon en: 1-Acuáticos: arroyos, pozas y charcas de agua, canaletas y vegetación sumergida; 2-saxícolas-terrestres: rocas, terraplenes y/o suelo; y 3-arborícolas: arbustos y troncos. En relación a los tipos de microhábitats utilizados por especie para cada tipo de vegetación, el ANOVA no paramétrico de Kruskal-Wallis mostró diferencias significativas para BTC ( $H$ $=21.4, P=0.007)$, BG $(H=47.8, P=0.001)$ y $\mathrm{P}(H=8.35, P=0.02)$, más no así para $\mathrm{MX}(H=10.1, P=$ 0.13). En la figura 4, se representan los modelos de diagramas de perfiles multivariados para los microhábitats usados por cada especie. El BTC, presentó una menor cantidad de microhábitats, al igual que el MX y P, si se le compara con el BG, con 13 de los 17 microhábitats utilizados por la comunidad de 
anuros. Las especies que utilizan un mayor número de microhábitats fueron L. neovolcanicus (9; principalmente pozas y charcas de agua, así como arroyos) A. compactilis (6; rocas en su mayoría y terraplenes), $D$. eximius (5; arbustos y rocas), e $H$. variolosus (4; charcas, pastos y rocas). En tanto, las que menos microhábitats utilizaron fueron $E$. guttilatus (2; arbustos), D, arenicolor (2; rocas y pastos), $S$. multiplicata (1; terraplenes), C. occidentalis (1, arbustos) e I. occidentalis (1; rocas) (Ver Fig. 4). El ANOVA no paramétrico de Kruskal-Wallis mostró diferencias significativas $(H=47.8, P=001)$ entre especies en relación al tipo de microhábitat utilizados. Por otra parte, el índice de solapamiento de nicho de Pianka $(O j k)$ presentó el valor más alto entre A. compactilis e I. occidentalis con 1, para I. occidentalis y D. arenicolor fue del $0.86 \%$, y en I. occidentalis y L. neovolcanicus del $0.72 \%$.

Cuadro 2. Periodos de actividad (hora promedio e intervalo) de las especies de la comunidad de anuros en el Área Natural Protegida Las Musas, Guanajuato. Nc = intervalo no considerado.

\begin{tabular}{lccc}
\hline Especies & $\begin{array}{c}\text { Hora de } \\
\text { actividad }\end{array}$ & Intervalo & $\begin{array}{c}\text { No. } \\
\text { Individuos }\end{array}$ \\
\hline Incilius occidentalis & $19: 43$ & $12: 45-23: 00$ & 5 \\
Anaxyrus compactilis & $16: 00$ & $10: 28-22: 10$ & 49 \\
Spea multiplicata & $21: 33$ & $\mathrm{Nc}$ & 1 \\
Craugastor occidentalis & $12: 50$ & $\mathrm{Nc}$ & 1 \\
Eleutherodactylus guttilatus & $10: 32$ & $\mathrm{Nc}$ & 2 \\
Dryophytes arenicolor & $15: 55$ & $11: 00-21: 46$ & 16 \\
Dryophytes eximius & $15: 29$ & $11: 20-22: 22$ & 56 \\
Hypopachus variolosus & $20: 20$ & $19: 20-22: 20-$ & 10 \\
Lithobates neovolcanicus & $18: 00$ & $10: 40-22: 20$ & 72 \\
\hline Total & $16: 56$ & $10: 28-23: 00$ & $N=212$ \\
\hline
\end{tabular}

Actividad. El ANOVA no paramétrico de Kruskal-Wallis mostró diferencias significativas $(H=$ 19.2, $P=0.018$ ) entre especies en relación a la hora de actividad. Asimismo, la prueba de igualdad de varianzas indicó diferencias significativas entre $A$. compactilis vs. $H$. variolosus $(F=9.05, P=0.014) ; D$. arenicolor vs. $H$. variolosus $(H=10.7, P=0.001)$, $D$. eximius vs. $H$. variolosus $(F=10.9, P=0.009), H$. variolosus vs. $I$. occidentalis $(F=0.08, P=0.007)$, y $H$. variolosus y $L$. neovolcanicus $(F=0.09, P=$ 0.004) (Cuadro 2). Respecto a la hora de actividad para las especies y entre tipos de vegetación, el ANOVA no paramétrico de Kruskal-Wallis, mostró diferencias significativas $(H=23.3, P=0.003)$ sólo para BG, en tanto que para los otros tipos de vegetación no ocurrió así (BTC, $H=7.6, P=0.10$; MX, $H=$ 01.5, $P=0.33$; $P, H=5.26, P=0.14$ ). Por otra parte, el análisis de regresión lineal mostró una relación positiva y significativa entre la temperatura cloacal con respecto a la temperatura del microhábitat $\left(\mathrm{T}^{\circ} \mathrm{CMH}\right)$, y la temperatura ambiente $\left(\mathrm{T}^{\circ} \mathrm{CA}\right)$ para $A$. compactilis $\left(\mathrm{T}^{\circ} \mathrm{CC}, r^{2}=0.74, P=0.001 ; \mathrm{T}^{\circ} \mathrm{CA}, r^{2}=\right.$ $0.68, P=0.01$ ), I. occidentalis $\left(\mathrm{T}^{\circ} \mathrm{CC}, r^{2}=0.95, P=0.005 ; \mathrm{T}^{\circ} \mathrm{CA}, r^{2}=0.82, P=0.02\right)$, D. eximius $\left(\mathrm{T}^{\circ} \mathrm{CC}\right.$, $\left.r^{2}=0.32, P=0.01 ; \mathrm{T}^{\circ} \mathrm{CA}, r^{2}=0.36, P=0.01\right)$ y $L$. neovolcanicus $\left(\mathrm{T}^{\circ} \mathrm{CC}, r^{2}=0.56, P=0.001 ; r^{2}=0.57, P\right.$ $=0.01$ ). En tanto que para $D$. arenicolor $\left(\mathrm{T}^{\circ} \mathrm{CC}, r^{2}=0.15, P=0.14\right.$; $\left.\mathrm{T}^{\circ} \mathrm{CA}, r^{2}=0.24, P=0.03\right)$, e $H$. variolosus $\left(\mathrm{T}^{\circ} \mathrm{CC}, r^{2}=0.44, P=0.23\right.$; $\mathrm{T}^{\circ} \mathrm{CA}, r^{2}=0.21, P=0.10$ ) no la hubo (Fig. 5 ).

El análisis de componentes principales (ACP) muestra una relación y variación entre especies en relación al uso del microhábitat y actividad. El ACP 1 explica una correlación del $49.1 \%$ en tanto que el ACP 2, el 20.4 \%, siendo la variación total explicada del 69.5 \%. EL ACP1 presentó una alta correlación para las variables $\mathrm{T}^{\circ} \mathrm{C} \mathrm{A}(0.91), \mathrm{T}^{\circ} \mathrm{C} \mathrm{MH}(0.92)$ y $\mathrm{T}^{\circ} \mathrm{CC}(0.84)$ en tanto que para el ACP 2, las variables altamente correlacionadas fueron el MH (85) y la VEG (0.61), con una correlación total de Ce $=0.911$ (Cuadro 3; Fig. 6). 
Cuadro 3. Análisis de componentes principales. Resultados de la correlación entre el microhábitat y actividad para la comunidad de anuros en el Área Natural Protegida Las Musas, Guanajuato. Variables: Microhábitat $=\mathrm{MH}$, temperatura del microhábitat $=\mathrm{T}^{\circ} \mathrm{CMH}$, temperatura ambiental $=\mathrm{T}^{\circ} \mathrm{CA}$, vegetación $=$ VEG, hora de actividad $=$ HORA, y temperatura cloacal $=\mathrm{T}^{\circ} \mathrm{CC}$.

\begin{tabular}{ccc}
\hline Variables & ACP 1 & ACP 2 \\
\hline HORA & -0.55 & 0.31 \\
MH & -0.27 & 0.85 \\
$\mathrm{~T}^{\circ} \mathrm{CA}$ & 0.91 & -0.02 \\
$\mathrm{~T}^{\circ} \mathrm{CMH}$ & 0.92 & 0.04 \\
$\mathrm{~T}^{\circ} \mathrm{CC}$ & 0.84 & 0.14 \\
VEG & -0.44 & -0.61 \\
\hline
\end{tabular}
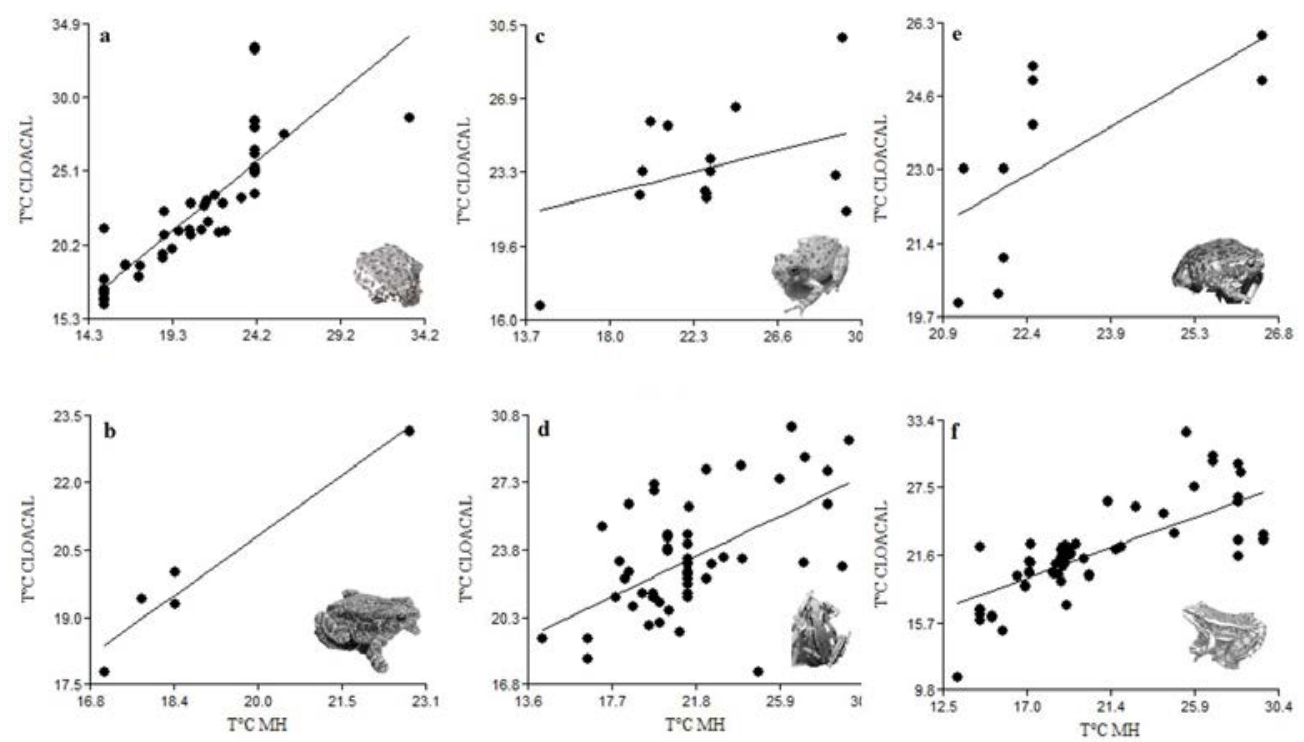

Figura 5. Regresión lineal entre temperatura corporal (cloacal) de seis especies de anfibios y la temperatura del microhábitat en el Área Natural Protegida Las Musas, Guanajuato; a = Anaxyrus compactilis, $\mathrm{b}=$ Incilius occidentalis, $\mathrm{c}=$ Dryophytes arenicolor, $\mathrm{d}=D$. eximius, $\mathrm{e}=$ Hypopachus variolosus y $\mathrm{f}=$ Lithobates neovolcanicus.

\section{DISCUSIÓN}

En ambientes estacionales tropicales los patrones de abundancia y distribución espacial de vertebrados como los anuros, son una respuesta directa a los periodos estacionales de lluvias y secas, por lo que es marcadamente mayor la abundancia en la estación de lluvias (Lajmanovich, 2000; Muñoz-Guerrero et al., 2007; Luja et al., 2016). Ello se plasma en el presente estudio en el que se observaron valores de abundancia altos para los meses de julio a septiembre durante la estación de lluvias pero sólo para cuatro de las especies; $L$. neovolcanicus, A. compactilis, D. eximius, y $D$. arenicolor, y en el caso de $H$. variolosus e I. occidentalis, sus abundancias fueron más bajas. (Fig. 3), lo que sin duda se relacionó con los ciclos reproductivos de las especies que coinciden con la temporada de lluvias, dando un incremento en su abundancia en función de la disponibilidad mayor de refugios y alimento (Ramírez-Bautista et al., 


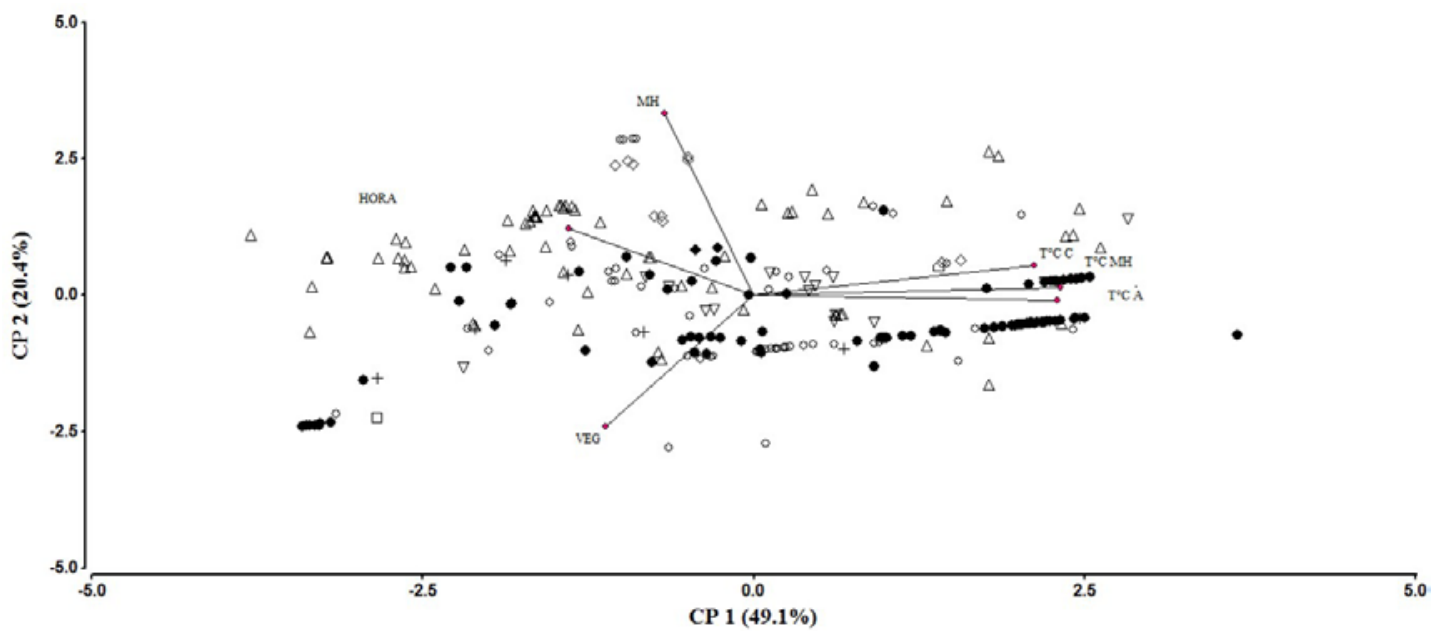

Figura 6. Análisis de componentes principales que muestra las variables altamente correlacionadas para las especies de la comunidad de anuros en función del microhábitat en el Área Natural Protegida Las Musas, Guanajuato. Las abreviaturas de las variables se encuentran en el Cuadro 3. Las especies se representan por los símbolos: $\diamond=$ Incilius occidentalis, $\Delta=$ Anaxyrus compactilis, $+=$ Spea multiplicata, $=$ Craugastor occidentalis, $\square=$ Eleutherodactylus guttilatus, $\nabla=$ Dryophytes arenicolor, $\bullet=$ Dryophytes eximius, $\mathbf{\Delta}=$ Hypopachus variolosus, $\mathrm{y}^{\circ}=$ Lithobates neovolcanicus.

2009). Sin embargo, no todas las especies fueron abundantes, ya que C. occidentalis, E. guttilatus y $S$. multiplicata, presentaron una abundancia muy baja y se registraron sólo durante la estación de lluvias. La baja abundancia de estas tres especies que pueden considerarse raras en el ANPLM y restringidas al BTC y $\mathrm{P}$, es una respuesta adaptativa a ambientes estresantes como los bosques tropicales caducifolios en los que el alimento y refugio (microhábitat) son limitados en estación de secas y tienen impacto sobre sus ciclos reproductivos y sobrevivencia (Luria-Manzano, 2012; Soto-Sandoval et al., 2017). Otro factor que puede explicar la baja abundancia, como en el caso particular de $S$. multiplicata, es el impacto del ganado vacuno (pastoreo) sobre los escasos sitios de refugio de la especie, siendo que se encontró sólo en el pastizal inducido con vestigios de bosque tropical caducifolio). Esta afectación sobre las condiciones de la cobertura vegetal nativa y su modificación a zonas de pastoreo, como ocurre en el área de estudio, ha sido reportado en otros trabajos en ambientes tropicales, observándose que la afectación sobre la vegetación nativa induce a una baja heterogeneidad de refugios, lo cual afecta la supervivencia y distribución vertical y horizontal de algunas especies con bajo espectro de distribución (Acuña-Vargas, 2016).

Microhábitats. La selección de los microhábitats por parte de los anuros en el sitio de estudio, podría estar determinada por la disponibilidad de éstos en cada tipo de vegetación (Fig. 4), así como por los periodos de actividad, su fisiología y capacidad de distribución y plasticidad para explotarlos (Navas 1996; Cuadro 2). Al respecto, encontramos que la especie con mayor distribución, abundancia y tipo de microhábitats utilizados fue $L$. neovolcanicus. Las especies de género Lithobates suelen considerarse de alta plasticidad al invadir y explotar variados ambientes, y los hábitos reproductivos asociados a charcas o pozas les brinda mayor independencia de fuentes de agua permanentes. Al respecto, Leivas et al. (2012) mencionan que $L$. catesbeianus presenta una alta plasticidad tanto trófica como en el uso de microhábitats, siendo una especie con un alto éxito ecológico y tolerante a distintos ambientes. Una situación similar podría ocurrir con $L$. neovolcanicus, ya que se observó con una amplia distribución y que a pesar de tener preferencia por pozas de agua aledañas al río Colorado y dentro del mismo río, usa una gran cantidad de microhábitats. Sin embargo, especies como C. occidentalis, S. multiplicata, E. guttilatus y D. arenicolor, presentaron una distribución limitada, a uno o dos tipos de vegetación, y en su caso no explotan más de 
tres microhábitats, lo que puede traducirse en la incapacidad de estas especies para invadir o colonizar nuevos ambientes, o bien pueden presentar restricciones de orden filogenético, lo que autores como Wiens y Graham (2005) denominan "conservadurismo del nicho”. Por ejemplo, se encontró que especies como C. occidentalis y E. guttilatus presentan una marcada ocurrencia sólo en la estación de lluvias además de una distribución restringida a BTC, por lo que puede considerarse que ambas especies están muy bien adaptadas a elementos vegetales del BTC, lo cual determina no sólo su presencia, sino también el uso de microhábitats. En contraste, otras especies del género Craugastor que habitan ambientes tropicales perennifolios, como el caso de C. loki, cuyo uso del microhábitat y distribución espacial está definida por sus estrategias reproductivas y puesta de los huevos (Urbina-Cardona \& Reynoso, 2010). Para especies como I. occidentalis y A. compactilis se observó una preferencia por microhábitats rocosos, principalmente en BG (Fig. 3).

Los sapos I. occidentalis y S. multiplicata, ambas de hábitos saxícolas-terrestres, se observaron principalmente en rocas y en terraplenes, y su escasa presencia se atribuye a la perturbación de su entorno. La primera, está limitada a una zona alterada (la zona de visitantes en BG), y durante el desarrollo del trabajo de campo se observó que los sitios que emplea como refugio fueron alterados por la remoción de rocas. En tanto que para $S$. multiplicata, en las zonas de pastoreo para ganado dónde se le encontró, se observó una baja abundancia. Por lo que las alteraciones al hábitat pueden influir tanto en la abundancia como en la selección del microhábitat, y en ese caso repercuten directamente sobre la sobrevivencia de las especies (Luja et al., 2016; Acuña-Vargas, 2016). El hylido D. arenicolor, se presentó principalmente en arroyos y río Colorado paralelos al BG, en microhábitats rocosos, en tanto que $D$. eximius, en BG y BTC, hace uso de once de los 17 microhábitats, en su mayoría relacionados con vegetación arbustiva circundante a los cuerpos de agua y zonas de alta humedad, $H$. variolosus, al igual que $D$. eximius se presentó en BG y BTC, asociado a microhábitats arbustivos en charcas y canaletas cercanas al río Colorado. El uso del microhábitat por las especies muestra que la comunidad de anuros, está integrada por tres grupos eco-estructurales que comprenden especies acuáticas (L. neovolcanicus), especies saxícolasterrestres (I. occidentalis, A. compactilis, S. multiplicata, D. arenicolor e $H$. variolosus), y especies arborícolas (D. eximius, C. occidentalis y E. guttilatus).

Solapamiento. Sólo en cuatro (L. neovolcanicus, A. compactilis, I. occidentalis y D. arenicolor) de las nueve especies ocurrió solapamiento. Aludimos este solapamiento a que las cuatro especies comparten hábitos similares, y sus periodos de actividad pueden coincidir entre sí (ver Cuadro 2), además la mayor parte de los registros y avistamientos fue en rocas, terraplenes y montículos de arena en BG y BTC. Por lo que la selección del microhábitat y la fluctuación de los valores de solapamiento entre especies, no dependerá solamente de la disponibilidad de refugios, sino también de la capacidad para enfrentar tolerancia fisiológica y conductual de cada especie (Wiens \& Graham, 2005; Roberto et al., 2011; RothMonzón et al., 2018).

Actividad. Los periodos de actividad diarios de la comunidad de anuros abarcan desde la mañana hasta la noche, a excepción de $H$. variolosus, cuya actividad ocurrió a partir de las 19:20 y hasta las 22:20 hrs (Cuadro 2). Este comportamiento tendría que ver con estrategias de termorregulación propias de cada especie como una respuesta a condiciones de temperatura en el ambiente (Sanabria et al., 2003). En este sentido, las especies en el ANPLM responden a dos estrategias de termorregulación que definen sus periodos de actividad. Especies termorreguladoras como D. arenicolor e I. occidentalis que mantienen la temperatura corporal esencialmente constante (Vitt \& Caldwell, 2014), durante los periodos diarios en los que se encuentran activas; en este caso, fueron las únicas en las que no se presentó una relación entre su temperatura cloacal con la del microhábitat y ambiental. En contraste, A. compactilis, D. eximius, $H$. variolosus y L. neovolcanicus, presentaron una relación con la temperatura del microhábitat y la ambiental (Fig. 5), por lo que se pueden considerar como especies termoconformistas, ya que si dependieron de las condiciones del ambiente (Huey \& Slatkin, 1976). Es decir, es posible que la preferencia de cada especie 
de acuerdo a los hábitats disponibles puede ser el reflejo de un rango eco-fisiológico de tolerancia en el ensamblaje de especies en la localidad.

El análisis de componentes principales (ACP) (Fig. 6), muestra que el comportamiento de la comunidad de anuros en relación a su estructura, distribución y selección de microhábitats, se explica por los periodos de actividad diarios, estrategias de termorregulación y hábitos que presentan las especies. En este contexto, se hipotetizó que la heterogeneidad vegetal, era el factor de respuesta a la selección del microhábitat y actividad de las especies. Sin embargo, no ocurrió así, puesto que las especies parecen responder a eventos de orden fisiológico y conductal en la selección de los microhábitats (Suazo-Ortuño et al., 2015), lo cual define su composición. En términos eco-estructurales, las especies se agrupan en especies acuáticas, saxícolas-terrestres y arbóreas, y en términos de sus necesidades energéticas, en especies termorreguladoras activas y termoconformistas. Finalmente, este trabajo es el primero en su tipo para el área de estudio y el estado de Guanajuato, y da la pauta para continuar con estudios puntuales sobre demografía de poblaciones y su monitoreo permanente, con énfasis en el desarrollo de estudios reproductivos a fin de tener información que permita en un futuro establecer el estado de conservación de los anuros en el sitio.

Agradecimientos. A los habitantes de la comunidad de Torrecilla y personal de vigilancia de la CONANP en el Área Natural Protegida Las Musas por su valioso apoyo durante el trabajo de campo. A Humberto González Parra, María Fernanda Rodríguez Gutiérrez, Carmen G. Mendoza Portilla, José Fonseca Padro y Rubí González García por su colaboración durante el trabajo de campo. Al Tecnológico Nacional de México por el apoyo económico brindado para el proyecto TNM-013-Convocatoria apoyo a la Investigación Científica, Aplicada, Desarrollo Tecnológico e Innovación 2016. A la Dirección General de Vida Silvestre (DGVS) de la Secretaría del Medio Ambiente y Recursos Naturales (SEMARNAT) por el otorgamiento del permiso de colecta científica SGPA/DGVS/06622/13) al primer autor (ALM). Finalmente, a los revisores que con sus comentarios enriquecieron la calidad del presente estudio.

\section{LITERATURA CITADA}

Acuña-Vargas, J. C. (2016) Anfibios y reptiles asociados a cinco coberturas de la tierra, Municipio de Dibulla, La Guajira, Colombia. Acta Zoológica Mexicana (nueva serie), 32, 133-146.

Balzarini M., Bruno C., Córdoba M., Teich, I. (2015) Herramientas en el Análisis Estadístico Multivariado. Escuela Virtual Internacional CAVILA. Facultad de Ciencias Agropecuarias, Universidad Nacional de Córdoba. Córdoba, Argentina. 200 pp.

Blanco Torres, A. (2009) Repartición de microhábitats y recursos tróficos entre especies de Bufonidae y Leiuperidae (Amphibia: Anura) en áreas con bosque seco tropical de la región Caribe-Colombia. Tesis de Doctorado, Universidad Nacional de Colombia.

Casas-Andreu, G., Valenzuela-López, G., Ramírez-Bautista, A. (1991) Cómo hacer una colección de anfibios y reptiles. Cuadernos del Instituto de Biología, UNAM, México, D.F., 68 pp.

Chave, J. (2013) The problem of pattern and scale in ecology: what have we learned in 20 years? Ecology Letters, 16, 1-13.

Gabriel, K. R. (1971) The biplot graphic display of matrices with application to principal components analysis. Biometrics, 58, 453-467.

García, E. (1973) Modificaciones al sistema de clasificación climática de Köppen: para adaptarlo a las condiciones de la República Mexicana. (Segunda edición). México, D.F., Universidad Nacional Autónoma de México. 246 pp.

García, M, A. Vera, C. J., Benetti, L. Blanco. (2016) Identificación y clasificación de los microhábitats de agua dulce. Acta Zoológica Mexicana (nueva serie), 32, 12-31. 
Huey, R. B., Slatkin, M. (1976) Cost and benefits of lizard thermoregulation. The Quarterly Review of Biology, 51, 363-384.

Juárez-Ortiz J., Eliosa-León, H. R., Gutiérrez-Mayén, M. A. (2016) Actividad acústica y efectos de la talla y factores ambientales sobre el canto nupcial de una comunidad de anuros de la Sierra Negra de Puebla. Pp. 133-148. In: Ma. G. Gutiérrez-Mayén, A, Ramírez-Bautista \& E. PinedaArredondo (Eds.). Ecología y conservación de anfibios y reptiles de México. Publicación especial No.4, Sociedad Herpetológica Mexicana, México.

Krebs C. J. (1999) Ecological methodology. Addison Wesley Longman. Second edition, NY, USA. 620 pp.

Lajmanochiv, R. C. (2000) Interpretación ecológica de una comunidad larvaria de anfibios anuros. Interciencia, 25, 71-79.

Leyte-Manrique, A., Morales-Castorena, J. P., Escobedo-Morales, L. A. (2016) Variación estacional de la herpetofauna en el cerro del Veinte, Irapuato, Guanajuato, México. Revista Mexicana de Biodiversidad, 87, 150-155.

Leivas, P. T., Leivas, F. W. T., Moura, M. O. (2012) Diet and trophic niche of Lithobates catesbeianus (Amphibia: Anura). Zoologia, 29, 405-412.

Lips, K. R., Mendelson III, J. R., Muñoz-Alonso, A., Canseco-Márquez, L., Mulcahy, D. G. (2004) Amphibian population declines in montane Southern Mexico: Resurveys of historical localities. Biological Conservation, 119, 555-564.

Luja V. H., Rodríguez-Estrella, R., Rojas-González, R. I. (2016) Reproducción y abundancia de la rana arborícola de Baja California Pseudacris hypochondriaca curta (Anura: Hylidae) en oasis de Baja California Sur. Pp. 76-100. In: Ma. G. Gutiérrez-Mayén, A, Ramírez-Bautista \& E. PinedaArredondo (Eds.). Ecología y conservación de anfibios y reptiles de México. Publicación especial No.4, Sociedad Herpetológica Mexicana, México.

Luria Manzano, R. (2012) Ecología trófica del ensamblaje de anuros riparios de San Sebastián Tlacotepec, Sierra Negra de Puebla, México. Tesis Maestría en Ciencias en Conservación. Universidad Autónoma del Estado de Hidalgo, Instituto de Ciencias Básicas e Ingeniera, Área Académica de Biología. Mineral de la Reforma Hidalgo. 80 pp.

Maciel-Mata, C. A., Manríquez-Moran, N., P. Octavio-Aguilar, P., Sánchez-Rojas, G. (2015) El área de distribución de las especies: Revisión del concepto. Acta Universitaria, 25, 3-19.

Muñoz-Guerrero, J., Serrano, V. H., Ramírez-Pinilla, M. P. (2007) Uso de microhábitat, dieta y tiempo de actividad en cuatro especies simpátricas de ranas hílidas neotropicales (Anura: Hylidae). Caldasia, 29, 413-425.

Navas, C. A. (1996) Implications of microhabitat selection and patterns of activity on the thermal ecology of high elevation Neotropical anurans. Oecologia, 108, 617-626.

Pérez-Tovar, R. L. (2011) Estructura y composición de un ensamblaje de anuros en un gradiente altitudinal de montaña perturbado en la secuencia del río Las Piedras (Popayán Cauca, Colombia). Trabajo de maestría en desarrollo sostenible y medio ambiente. Universidad de Manizales. $94 \mathrm{pp}$.

Pianka, E. R. (1986) Ecology and natural history of desert lizards: Analyses of the ecological niche and community structure. Copeia, 1986, 1033-1036.

Pounds A., Fogden, M., Campbell, J. (1999) Biological response to climate change on a tropical mountain. Nature, 398, 611-614.

Ramírez-Bautista, A., Hernández-Salinas, U., García-Vázquez, O., Leyte-Manrique, A., CansecoMárquez, L. (2009) Herpetofauna del Valle de México: Diversidad y conservación. Universidad Autónoma del Estado de Hidalgo y Comisión Nacional para el Conocimiento y Uso de la Biodiversidad. México, D.F. 213 pp.

Roberto, I. J., Brito L., Cascon, P. (2011) Temporal and spatial patterns of reproductive activity in Rhinella hoogmoedi (Anura: Bufonidae) from a tropical rainforest in northeastern Brazil, with the description of it's advertisement call. South American Journal of Herpetology, 6, 87-97. 
Roth-Monzón, A. J., Mendoza-Hernández, A. A., Flores-Villela, O. (2018) Amphibian and reptiles biodiversity in the semi-arid region of the municipality of Nopala de Villagrán, Hidalgo, Mexico. PeerJ, 4202, 3-21.

Rzedowski, J. (1978) Vegetación de México. México, D.F. Limusa. 431 pp.

Sanabria, E. A., Quiroga, L. B., Acosta, J. C. (2003) Relación entre la temperatura corporal de adultos de Bufo arenarum (Anura: Bufonidae) y variables ambientales en un humedal de San Juan, Argentina. Multequina, 2, 49-53.

Soto Sandoval, Y., Suazo-Ortuño, I., N. Urbina-Cardona, N., J. Marroquín-Páramo, J., AlvaradoDíaz, J. (2017) Efecto de los estadios sucesionales del bosque tropical seco sobre el microhábitat usado por Agalychnis dacnicolor (Anura: Phyllomedusidae) y Smilisca fodiens (Anura: Hylidae). Revista de Biología Tropical, 65, 777-798.

Suazo-Ortuño, I., Alvarado-Díaz, J., Mendoza, E., López-Toledo, L., Lara-Uribe, N., MárquezCamargo, C., Rangel-Orozco, J. D. (2015) High resilience of herpetofaunal communities in a human-modified tropical dry forest landscape in western Mexico. Tropical Conservation Science, 8, 396-423.

Urbina-Cardona, J. N., Reynoso, V. H. (2010) Uso del microhábitat por hembras grávidas de la rana de hojarasca Craugastor loki en la selva alta perennifolia de Los Tuxtlas, Veracruz, México. Revista Mexicana de Biodiversidad, 80, 571-573.

Vargas-S. F., Bolaños, M. E. L. (1999) Anfibios y repties presentes en hábitats perturbados de la selva lluviosa tropical en el Bajío Anchicayá, Pacifico Colombiano. Revisión Académica Colombiana Científica. Vol. XXIII Suplemento Especial. 13 pp.

Vasconcelos, T.S., Rosas-Feres, D. C. (2008) Habitat heterogeneity and use of physical and acoustic space in anuran communities in Southeastern Brazil. Phyllomedusa, 7: 127-142.

Vázquez-Díaz, J., Quintero-Díaz, G. E. (2005) Anfibios y reptiles de Aguascalientes. 2da. Ed. Comisión Nacional para el Conocimiento y Uso de la Biodiversidad, y Centro de Investigaciones y Estudios Multidisciplinarios de Aguascalientes, A. C. México, D. F. 318 pp.

Vitt, L., Caldwell, J. (2014) Herpetology an introductory biology of amphibians and reptiles. Academic Press- Elsevier, San Diego. 757 pp.

Vite-Silva, V. D., Ramírez-Bautista, A., Hernández-Salinas, U. (2010) Diversidad de anfibios y reptiles de la Reserva de la Biosfera Barranca de Metztitlán, Hidalgo, México. Revista Mexicana de Biodiversidad, 80, 473-485.

Walter, F., Brooks, B. (2009) Diagnóstico pesquero y acuícola de Guanajuato. Gobierno del Estado de Guanajuato, SAGARPA y Universidad Michoacana de San Nicolás de Hidalgo. 153 pp.

Wiens, J. J., Graham, C. H. (2005) Niche conservatism: Integrating evolution, ecology and conservation biology. Annual Reviews Ecology, Evolution and Systematic, 36, 519-39.

Williams-León de Castro, M., D. Rey-Sánchez. (2014) Primer registro de la presencia y reproducción del anuro Rhinella poeppigii (Tschudi, 1845) en un área antrópica del departamento de Lima, Perú. Ecología Aplicada, 13, 109-115.

Zar, J. H. (1999) Biostatistical analysis. Prentince-Hall, New Jersey. 994 pp. 